OPEN ACCESS

Edited by:

Nobuhiro Suzuki,

Sophia University, Japan

Reviewed by:

Tsung-Luo Jinn,

National Taiwan University, Taiwan

Stefan Simm,

Universitätsmedizin Greifswald,

Germany

*Correspondence:

Anthony Guihur

anthony.guihur@unil.ch;

anthony.guihur@gmail.com

Specialty section:

This article was submitted to

Plant Abiotic Stress,

a section of the journal

Frontiers in Plant Science

Received: 17 May 2021

Accepted: 06 July 2021

Published: 09 August 2021

Citation:

Bourgine B and Guihur A (2021)

Heat Shock Signaling in Land Plants:

From Plasma Membrane Sensing to the Transcription of Small Heat

Shock Proteins.

Front. Plant Sci. 12:710801. doi: 10.3389/fpls.2021.710801

\section{Heat Shock Signaling in Land Plants: From Plasma Membrane Sensing to the Transcription of Small Heat Shock Proteins}

\author{
Baptiste Bourgine and Anthony Guihur* \\ Department of Plant Molecular Biology, Faculty of Biology and Medicine, University of Lausanne, Lausanne, Switzerland
}

Heat stress events are major factors limiting crop productivity. During summer days, land plants must anticipate in a timely manner upcoming mild and severe temperature. They respond by accumulating protective heat-shock proteins (HSPs), conferring acquired thermotolerance. All organisms synthetize HSPs; many of which are members of the conserved chaperones families. This review describes recent advances in plant temperature sensing, signaling, and response. We highlight the pathway from heat perception by the plasma membrane through calcium channels, such as cyclic nucleotide-gated channels, to the activation of the heat-shock transcription factors (HSFs). An unclear cellular signal activates HSFs, which act as essential regulators. In particular, the HSFA subfamily can bind heat shock elements in HSP promoters and could mediate the dissociation of bound histones, leading to HSPs transcription. Although plants can modulate their transcriptome, proteome, and metabolome to protect the cellular machinery, HSP chaperones prevent, use, and revert the formation of misfolded proteins, thereby avoiding heat-induced cell death. Remarkably, the HSP2O family is mostly tightly repressed at low temperature, suggesting that a costly mechanism can become detrimental under unnecessary conditions. Here, the role of HSP2Os in response to HS and their possible deleterious expression at non-HS temperatures is discussed.

\footnotetext{
Keywords: heat shock transcription factor, heat shock response, acquired thermotolerance, cyclic nucleotidegated channels, calmodulins, small heat-shock proteins, heat stress, global warming
}

\section{INTRODUCTION}

During summer days, mild or severe heat stress (HS) typically occurs at midday and lasts until late afternoon in terrestrial systems (Dong et al., 2017). To survive, a plant must sense, early in the morning, a minor temperature increment to establish a suitable genetic program. The heat shock response (HSR) contains molecular defenses, including heat shock proteins (HSPs), that

Abbreviations: ARP6, actin-related protein 6; AT, acquired thermotolerance; CaM, calmodulin; CaMBD, calmodulinbinding domain; CBK3, calmodulin-binding protein kinase 3; CML, calmodulin-like protein; CNBD, cyclic nucleotidebinding domain; CNGC, cyclic nucleotide-gated channels; ER, endoplasmic reticulum; IQ, isoleucine-glutamine; HS, heat stress; HSE, heat shock element; HSF1, heat shock transcription factor; HSP, heat shock protein; HSR, heat shock response; miRNAs, microRNAs; ncRNA, non-encoding RNA; ROS, reactive oxygen species; siRNAs, small interfering RNAs. 
must accumulate rapidly under rising temperatures to minimize foreseeable damage (Song et al., 2012; Serrano et al., 2019). Acquired thermotolerance (AT) refers to the plant adaptive capacity to survive noxious HS when exposed to sublethal temperatures, requiring the accumulation of HSPs. Under HS, both transcriptome and proteome-based studies have indicated regulatory responses of HSPs (Qin et al., 2008; Finka et al., 2011; Mangelsen et al., 2011; Xin et al., 2016; Guihur et al., 2020; Zhao et al., 2021). A conserved subfamily called "heat-induced molecular chaperones" contains the HSP100s, HSP90s, HSP70s, HSP60s, HSP40s, and HSP20s (Al-Whaibi, 2011; Jee, 2016). They are 20 times more likely to be heat-induced compared to nonchaperone proteins (Wang et al., 2004; Finka et al., 2011, 2015; Guihur et al., 2020). HSP chaperones prevent and repair protein misfolding and aggregation, reducing cell damage (Ben-Zvi and Goloubinoff, 2001; Wang et al., 2004; Zeng et al., 2004; Liberek et al., 2008; Mogk and Bukau, 2017). In particular, HSP20s are the most heat-responsive in plants due to their dramatic induction (Vierling, 2003; Guihur et al., 2020). They also prevent the aggregation of heat-labile proteins and could stabilize lipids at the plasma membrane (Haslbeck and Vierling, 2015). At low temperature, HSP20s are tightly repressed, suggesting that their inappropriate expression could be deleterious for plants (Sun et al., 2016). Moreover, HS generates stress granules that contain molecular chaperones, such as HSP20s, HSP101, untranslated mRNAs, elongation initiation factors, RNA-binding proteins and transcription factors (McLoughlin et al., 2016, 2019; Chantarachot and Bailey-Serres, 2018; Kosmacz et al., 2019). Theses cytoplasmic and chloroplastic bodies seem to have an important role in protein translation during and after HS (Merret et al., 2017; Chodasiewicz et al., 2020).

In most eukaryotes, including land plants, HSP accumulation depends on a signal that arises at the plasma membrane and results in the activation of heat shock transcription factor (HSF) families (Nover et al., 2001; Mishra et al., 2002; Hayashida et al., 2011; Liu et al., 2011; Scharf et al., 2012; Fragkostefanakis et al., 2015; Kijima et al., 2018). Plant cells can sense a wide temperature range through changes in the plasma membrane fluidity. Calcium channels, such as cyclic nucleotide-gated channels (CNGCs), can mediate calcium entry during HS as shown in Arabidopsis thaliana and Physcomitrium patens (Gong et al., 1998; Saidi et al., 2009; Finka et al., 2012; Gao et al., 2012; Tunc-Ozdemir et al., 2013). Yet, a fraction of HSFA1 is associated with the HSP70HSP90 complex, and a large inactivated fraction might remain unbound under non-stressful conditions (Kyle Hadden et al., 2006; Westerheide et al., 2006; Saidi et al., 2009; Hahn et al., 2011). Following the activation of CNGCs, and a still unclear signaling pathway, HSFA1 is translocated into the nucleus and can bind specific DNA motifs present in the promoter of HSP genes, called "heat shock element" (HSE) (Santoro et al., 1998; Liu and Charng, 2012). HSFA1 could also trigger regulatory responses, including DNA methylation, histone modification, and chromatin remodeling (Zhao et al., 2021). In particular, bound histones to HSP genes, such as H2A.Z, must be evicted to allow for RNA polymerase II docking for the transcription of HSP (Franklin, 2010; Kumar and Wigge, 2010; Probst and Mittelsten Scheid, 2015). Yet, the mechanisms of heat sensing, particularly the components between the CNGCs sensors and the activation of the main regulator HSFA1 are not elucidated (Larkindale et al., 2005; Mittler et al., 2012). This review addresses the heat perception and signaling pathway in land plants, with a particular emphasis on the activation of HSFA1 at the plasma membrane, leading to the accumulation of HSP chaperones. In addition, the role of HSP20s at non-HS temperatures and their putative effect in plant cells are discussed. Understanding these critical processes would facilitate the production and selection of thermotolerant cultivars to face global warming.

\section{HEAT SENSING AND SIGNALING IN LAND PLANTS}

\section{Calcium Entry Across the Plasma Membrane Triggers the Heat Shock Signaling}

Plant cells developed an efficient mechanism for sensing the increase in temperature, as well as a signaling cascade for a rapid adaptive response. The nucleus, endoplasmic reticulum, cytosol, mitochondria, and chloroplast may also contain heat sensors (Bussell et al., 2010; Franklin, 2010; Schwarzländer and Finkemeier, 2012; Hentze et al., 2016; Sun and Guo, 2016; Chang et al., 2017; Lin K. F. et al., 2018). However, various observations have indicated that the primary heat sensing might occur at the plasma membrane. For instance, electrophysiology measurements in $P$. patens protoplasts, expressing the calcium-sensitive fluorescent protein aequorin reporter, have demonstrated a saturated accumulation of cytosolic $\mathrm{Ca}^{2+}$ within the first $10 \mathrm{~min}$ at $38^{\circ} \mathrm{C}$ (Saidi et al., 2009). Artificially preventing the entry of periplasmic $\mathrm{Ca}^{2+}$ in $A$. thaliana and $P$. patens protoplast showed a lack of HSP expression. A defective HSR has also been described in the presence of ionomycin and thapsigargin, which are ionophores known to release $\mathrm{Ca}^{2+}$ from internal stores (Saidi et al., 2009; Finka et al., 2012). Similar observations were reported for tobacco, maize, and rice (Gong et al., 1998; Li et al., 2004; Wu and Jinn, 2010; Wu et al., 2012). Yet, this phenotype has not been observed in Chlamydomonas reinhardtii, suggesting another mechanism of heat perception in green algae (Schmollinger et al., 2013). Thus, the HSR seems to depend on $\mathrm{Ca}^{2+}$ entry across the plasma membrane in land plants (Figure 1; Demidchik et al., 2018).

\section{Embedded Cyclic Nucleotide-Gated Channels Act as Plasma Membrane Thermosensors}

In both plants and animals, plasma membrane contains CNGCs, which are tetrameric cation channels and contain six transmembrane domains. They modulate $\mathrm{Ca}^{2+}$ entry from the apoplast and other ions, such as $\mathrm{Mg}^{2+}, \mathrm{K}^{+}, \mathrm{Na}^{+}$, or $\mathrm{Pb}^{+}$(Véry and Sentenac, 2002). CNGCs can be assembled as homotetrameric or heterotetrameric complexes, allowing for the formation of a large array of sensors capable of responding to different intensities of environmental cues (Clough et al., 2000; Tan et al., 2020). The cytosolic C-terminus harbors a 
cyclic nucleotide-binding domain (CNBD) and a calmodulinbinding domain (CaMBD), in which a calmodulin (CaM) binding isoleucine-glutamine (IQ) motif is embedded (Kaplan et al., 2007; Jarratt-Barnham et al., 2021). A. thaliana AtCNGC2 and four acts as a heat sensor since its lack of expression leads to a hyper thermosensitive phenotype, resulting in a higher accumulation of HSPs at lower temperature. Similar results were observed in the orthologs CNGCb and CNGCd of P. patens (A Finka et al., 2012; Finka and Goloubinoff, 2014). In addition, the lack of AtCNGC6 in A. thaliana leads to a fewer transcript levels of HSP18.2, HSP25.3, and HSP70 compared to control plants at $37^{\circ} \mathrm{C}$, ultimately impacting the HSR (Gao et al., 2012). In rice, reduced thermotolerance was observed in Oscngc14 and Oscngc16 mutants, resulting in a higher accumulation of hydrogen peroxide, leading to the cell death (Cui et al., 2020). These results strongly suggest that CNGCs act as thermosensors in land plants. Other calcium channel families have been also implicated in heat response. For example, the lack of synaptotagmin A activity led to decreased HSPs synthesis in A. thaliana at $45^{\circ} \mathrm{C}$ (Yan et al., 2017). ANNEXIN calcium channels can modulate cytosolic calcium signature under oxidative and heat stresses (Liao et al., 2017). Glutamate receptorlike channels have been suggested to participate in $\mathrm{Ca}^{2+}$ signaling since exogenous glutamate improves basal thermotolerance in maize ( $\mathrm{Li}$ et al., 2019). Therefore, the heat sensing by the plasma membrane of plants contains calcium channels, including CNGCs, that can respond to incremental temperatures, mediating $\mathrm{Ca}^{2+}$ entry and triggering the signaling pathway for the accumulation of HSPs.

\section{Calmodulins Response to Heat}

The signaling molecules located in the cytosol and required to activate HSFA1s are not yet uncovered (Figure 1). Yet, both CNBD and CaMBD present on the cytosolic part of CNGCs suggest that cyclic nucleotide monophosphate and CaMs can mediate the heat signaling (Gao et al., 2012). CaMs are made of calcium-binding loops, called "E" and " $F$ " that can each bind two $\mathrm{Ca}^{2+}$ ions and can respond to biotic and abiotic stress in plants (Rhoads and Friedberg, 1997; Mccormack et al., 2005; Fischer et al., 2013; Virdi et al., 2015). A. thaliana contains nine CaMs; among them are the first seven, which are highly conserved. In addition, 50 members of calmodulin-like proteins (CMLs) have been described as important players in stress perception and plant development (Aldon and Galaud, 2006; Vadassery et al., 2012). Interestingly, AtCaM2, AtCaM4, AtCaM6, AtCaM7, and AtCML8 were found to bind the C-terminal of several CNGC families (Fischer et al., 2017). At $37^{\circ} \mathrm{C}$, AtCNGC6 was negatively regulated by AtCaM2/3/5 and AtCaM7, which interact with the IQ motif of AtCNGC6 and impact $\mathrm{Ca}^{2+}$ entry (Niu et al., 2020). A potentially important role in the heat signaling has been demonstrated for AtCaM3 where the knockout mutant has shown reduced levels of HSP18.2 and HSP25.3 transcripts at $37^{\circ} \mathrm{C}$, negatively impacting basal thermotolerance. The overexpression of AtCaM3 leads to a significant increase in the HSPs level and improves the resistance against noxious temperatures (Zhang et al., 2009). AtCaM3 has been also suggested to activate several components of the heat shock signaling pathway, such as mitogen-activated protein kinase 6 and calmodulin-binding protein kinase 3 (CBK3) (Figure 1; Liu et al., 2005; Yan et al., 2017). Moreover, AtCBK3 promotes HSFA1 activation by phosphorylation. Under HS, the lack of AtCKB3 dramatically reduced HSP18.2 and HSP25.3 levels, resulting in defective basal thermotolerance, whereas the Atcbk3 overexpression line rescued the hypersensitivity phenotype (Liu et al., 2008; Yip Delormel and Boudsocq, 2019). In other plant species, several CaMs have been described to mediate the heat signal. In rice, OsCaM1-1 was shown to positively regulate $\mathrm{Ca}^{2+}$ signals, resulting in HSP accumulation (Wu and Jinn, 2012; Wu et al., 2012). In wheat, CaM1-2 has been found to act upstream of HSP26 and HSP70 at $37^{\circ} \mathrm{C}$ (Liu et al., 2003). Therefore, CaMs have been mentioned to play a critical role in the heat signaling of land plants and responding to other environmental stimuli (Virdi et al., 2015). Yet, other components of the heat signaling pathway between CNGC sensors and HSFA1s remain to be identified (Figure 1).

\section{Heat Shock Transcription Factor A1 Acts as a Key Regulator of the Heat Shock Signaling Pathway}

HSFs are essential regulators of the heat signaling pathway in many organisms (Gallo et al., 1993; Mishra et al., 2002; Nicholls et al., 2009; Anckar and Sistonen, 2011). In contrast to vertebrates, which contain fewer members (six for humans), plant HSF families have more members that reflect their strategy for a sessile adaptation in changing environment (von KoskullDoring et al., 2007; Huang et al., 2016; Gomez-Pastor et al., 2018). For example, A. thaliana contains $21 \mathrm{HSFs}, 24$ for tomato, 52 for soybean, or 56 for wheat (Scharf et al., 2012; Xue et al., 2014; Fragkostefanakis et al., 2015). Plant HSFs are classified into three classes: HSFA, B, and C. All HSFs have a DNA binding, oligomerization, and nuclear localization domains. Yet, HSFAs differ in the presence of an activator region at the C-terminal, which binds HSEs, whereas HSFBs contain an inhibitor region. Under HS, several subfamilies of HSFA are required for the accumulation of HSPs. HSFB contains subfamilies, which can act as coactivators of HSPs transcription and, also, as antagonist repressors, competing for HSFAs at the end of HS (Czarnecka-Verner et al., 1997; Santoro et al., 1998; Mishra et al., 2002; Mitsuda and Ohme-Takagi, 2009; Ikeda et al., 2011; Scharf et al., 2012; Liu and Charng, 2013; Fragkostefanakis et al., 2015; Guo et al., 2016). In A. thaliana, the hsfAla mutant was shown to be ineffective in accumulating several HSP transcripts at $37^{\circ} \mathrm{C}$, resulting in the absence of AT (Liu et al., 2011). Similar observations were previously made in tomato where HSFA1 has been shown to be a master regulator for AT (Mishra et al., 2002). Studies in several monocotyledonous species, such as wheat and rice, have also demonstrated the important role of HSFA in mediating the heat signal (Yokotani et al., 2008; Zhang et al., 2013; Guo et al., 2016). Furthermore, the role of HSFA1 is not only limited to the transcription of HSPs, but it also activates several transcription factors, such as HSFA2, HSFA3, HSFA7a, multiprotein bridging factor 1C, and dehydration-responsive element-binding protein $2 \mathrm{~A}$, which are 


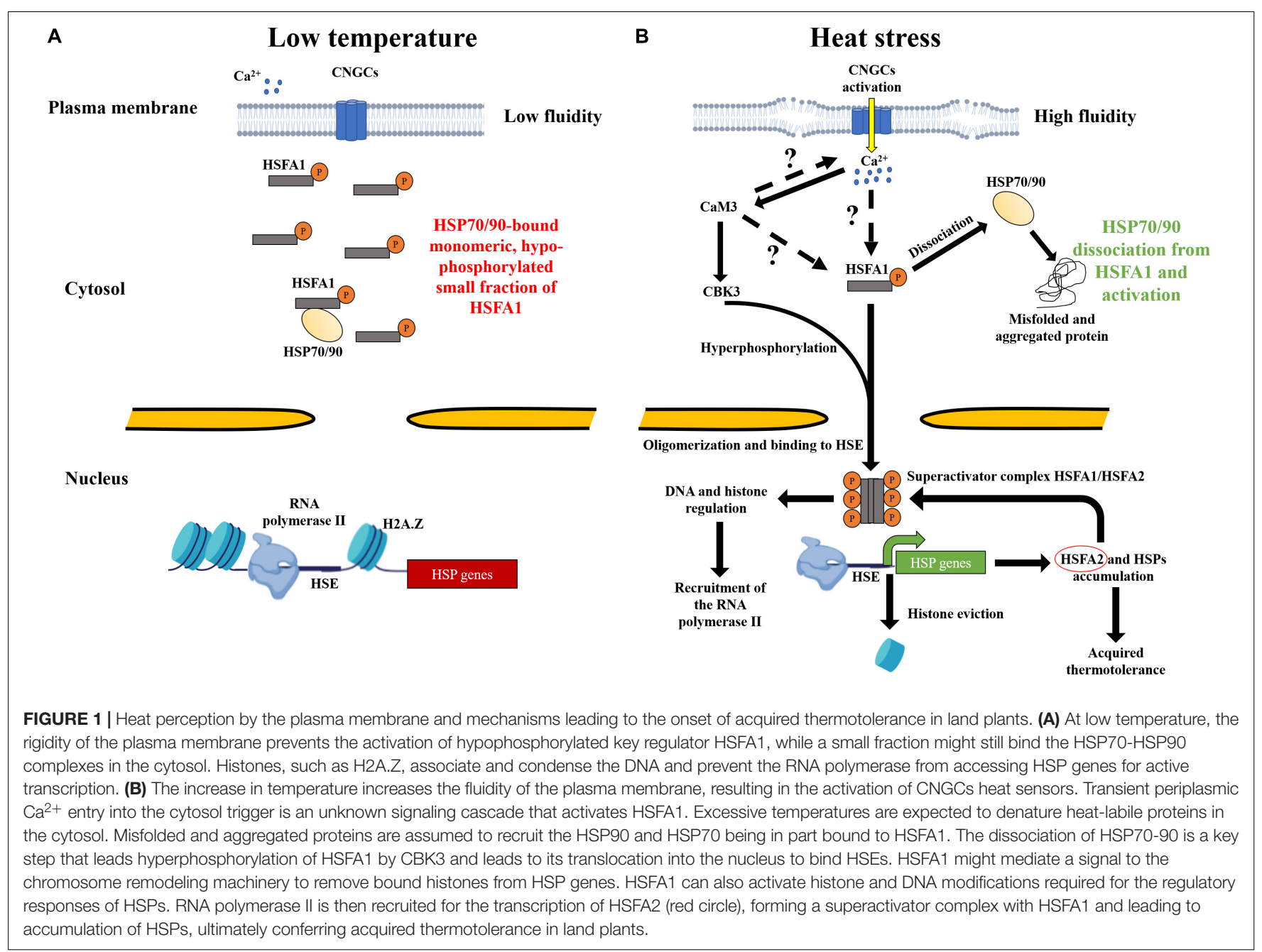

required for HSP synthesis and thermotolerance in A. thaliana (Suzuki et al., 2011; Yoshida et al., 2011; Liu and Charng, 2013; Ohama et al., 2017). When accumulated, HSFA2 can form a heterodimer with HSFA1 and thereby forming a superactivator complex for sustaining HSPs expression under HS (ChanSchaminet et al., 2009). Thus, the family of HSFAs has been identified as a major regulator required for the onset of AT in land plants (Mishra et al., 2002; Hahn et al., 2011; Yoshida et al., 2011).

At low temperature, inactive cytosolic HSFA1s are hypophosphorylated and bound to the complex HSP70HSP90 (Figure 1; Hahn et al., 2011; Morimoto, 2012). The traditional model suggests that, upon HS, HSP70-HSP90 complex is hijacked by the increased cytosolic levels of unfolded or misfolded thermolabile proteins, leaving HSFA1 free to trigger the HSR (Figure 1; Zou et al., 1998; Kim and Schöffl, 2002; Yamada et al., 2007; Hahn et al., 2011). Although thermolabile proteins become denatured and recruit molecular chaperones upon heat exposure, the prevention of $\mathrm{Ca}^{2+}$ entry through the plasma membrane led to an absence of HSR (Saidi et al., 2009). In addition, treatment with HSP90 inhibitors triggers a minor HSR at low temperature at rest, whereas a full-blown HSR can be obtained at higher temperatures (Kyle Hadden et al., 2006;
Westerheide et al., 2006; Saidi et al., 2009). Therefore, even if all HSP90s are dissociated from HSFA1s, a large fraction of HSFA1s is required to be activated independently from a non-elucidated signal, which strictly depends on the calcium entry across the plasma membrane (Figure 1).

\section{Histones and DNA Regulation Lead to the Transcription of HSPs}

The expression of HSPs in plants is regulated by epigenetics, such as DNA methylation, histone modification, and chromatin remodeling (Boyko et al., 2010; Gao et al., 2014; GallegoBartolomé, 2020; Zhao et al., 2021). HSPs gene must be unwrapped from histones to become actively transcribed when HSF1As are bound to HSEs (Figure 1). Following HS, a global rearrangement of the chromatin has been observed in rice, rye, and A. thaliana (Santos et al., 2011; Tomás et al., 2013; Probst and Mittelsten Scheid, 2015). These results indicate that, at low temperature, HSPs genes are compacted by histones, and chemical modifications are required for HSPs expression to be allowed under HS. Interestingly, in A. thaliana, actinrelated protein 6 (ARP6) has been reported to be an essential 
component of the chromatin remodeling complex required for H2A.Z incorporation (Figure 1; March-Díaz and Reyes, 2009). The Atarp6 mutant exhibited a higher accumulation of HSP70 at 12,22 , and $27^{\circ} \mathrm{C}$ (Kumar and Wigge, 2010; Cortijo et al., 2017). Histone chemical modifications, such as methylation and acetylation, have been also indicated to be important for the regulation of HSPs in plants (Lämke et al., 2016; Yamaguchi et al., 2021). Regarding non-coding RNA (ncRNA), such as microRNAs (miRNAs) and small interfering RNAs (siRNAs), its involvement in the regulation of the HSR has been suggested in several land plant species (Khraiwesh et al., 2012; Li et al., 2014; Zhao et al., 2016; Liu et al., 2017; Lin J. S. et al., 2018; Zhao et al., 2021). Thus, following a short period of HS, epigenetic processes have also been implicated in developing a transcriptional heat memory (Bäurle and Trindade, 2020).

\section{THE ROLE OF SMALL HEAT SHOCK PROTEINS UNDER HEAT STRESS}

Among HSP chaperones, the HSP20 (sHSP) family is the most responsive to heat, whereas, at low temperatures, it is mainly repressed (Waters et al., 1996; Sun et al., 2002; Vierling, 2003; Guihur et al., 2020). HSP20s proteins are composed of subunits between 12 and $43 \mathrm{kDa}$ and have an alpha-crystalline domain suggested to bind denatured proteins (Haslbeck et al., 2005; Basha et al., 2012; Waters and Vierling, 2020). A. thaliana contains 19 HSP20 (sHSPs) divided into six classes according to their localization (Sun et al., 2002; Waters and Vierling, 2020).
Following HS, heat-labile proteins can be bound by HSP20s, in an ATP-independent manner, and folded into their native state by chaperone machinery, thereby preventing further heat-caused denaturation (Waters et al., 1996; Glover and Lindquist, 1998; Veinger et al., 1998; Goloubinoff et al., 1999; Swindell et al., 2007; Haslbeck and Vierling, 2015; Mogk et al., 2015; Mogk and Bukau, 2017). As shown initially in E. coli, the small HSP IbpB has been revealed to interact with HSP40, HSP60, and HSP70 chaperone complexes and assist in protein refolding (Veinger et al., 1998). Similar observations have been made in Pisum sativum and Synechocystis sp. (Mogk et al., 2003). In addition, HSP20s stabilize lipid bilayers and thereby protect the plasma membrane from high fluidity under excessive temperatures (Horváth et al., 2008; Haslbeck and Vierling, 2015).

HSP20s accumulation is essential for basal thermotolerance and the onset of AT in plants. In A. thaliana, an AtHSP17.6II mutant was unable to establish the AT, whereas the overexpression of LimHSP16.45 from Lilium davidii rescued the sensibility to HS (Yang et al., 2020). Supporting these observations, A. thaliana RNAi lines of six cytosolic HSP20s showed higher thermosensitivity, whereas the HSP20s overexpression lines restored the phenotype (McLoughlin et al., 2016). In wheat, chloroplastic HSP26 was shown to be required for seed maturation, germination, and development of HS tolerance (Chauhan et al., 2012). Similar observations have been described in other plant species, such as in tobacco and rice (Lee et al., 2000; Zhang et al., 2016). Besides providing protection against noxious temperatures, HSP20s also confer resistance to salt, drought, and cold stresses (Sun et al., 2002;
A

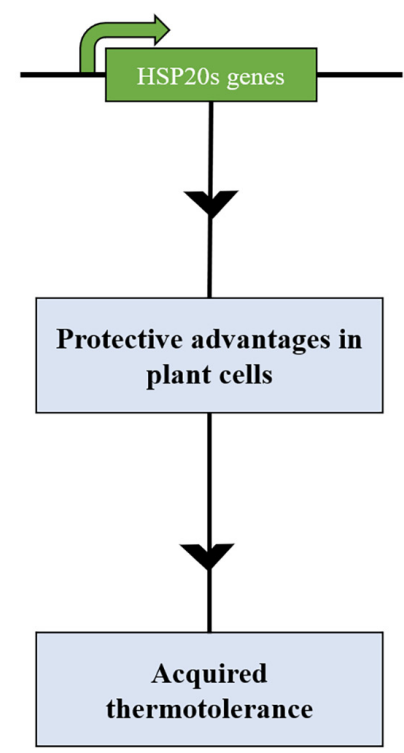

B

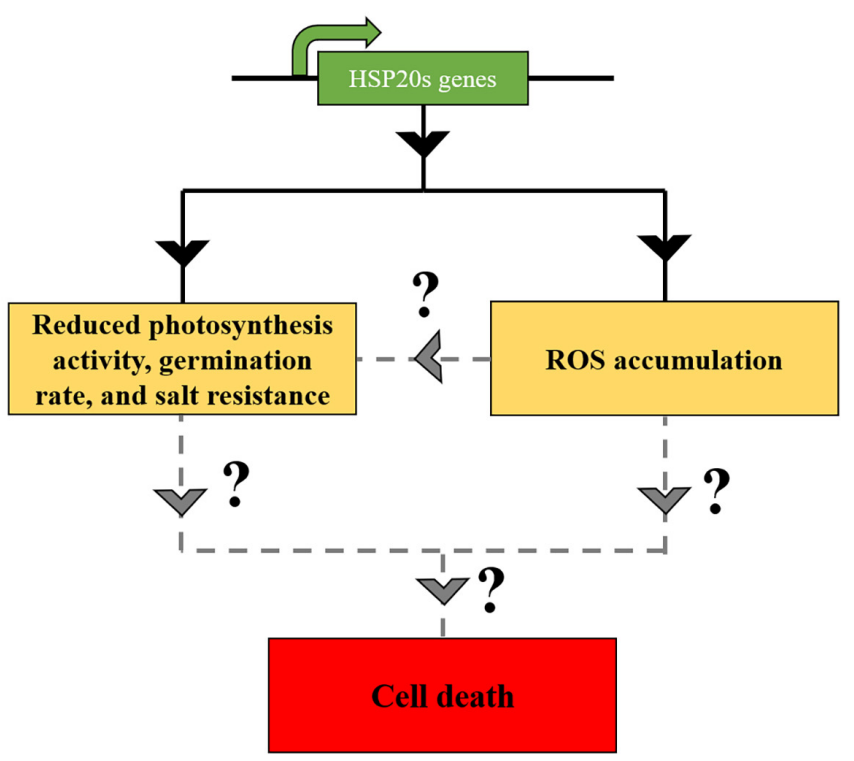

FIGURE 2 | The expression of HSP20s at low temperature might induce deleterious effects in land plants. (A) Under heat stress, HSP20s are accumulated, conferring protective advantages in plant cells, leading to the onset of acquired thermotolerance. (B) The overexpression of HSP20s at low temperatures might induce deleterious effect on plant growth and development (Sun et al., 2016), such as ROS accumulation (Ma et al., 2019), eventually resulting in the cell death (Sun et al., 2016; Ma et al., 2019). Solid lines indicate the consequences of HSP20s expression at low temperature, whereas dashed lines indicate potential cell death effect on plant cells. 
Sarkar et al., 2009; Song and Ahn, 2010; Yang et al., 2014). HSP20s were also described to play key roles in somatic embryogenesis, pollen development, and seed germination (Sun et al., 2002; Volkov et al., 2005; Chauhan et al., 2012).

In several plant species, transcriptome and proteome-based analyses have demonstrated a nearly total absence of HSP20s at non-HS temperatures (Hernandez and Vierling, 1993; SimõesAraújo et al., 2003; Finka et al., 2012; Guihur et al., 2020). In contrast, other HSP chaperones families might have a substantial constitutive expression (Finka et al., 2012; Guihur et al., 2020). This raises a question of why plants tightly suppress HSP20s synthesis at non-HS temperature (low temperature) The complete HSP20 repression suggests that its constitutive expression would be problematic (Figure 2). To date, one study has reported a deleterious effect of one HSP20 in A. thaliana. A. stolonifera HSP17 overexpression in A. thaliana led to a reduction in leaf chlorophyll content and photosynthesis activity at both 22 and $40^{\circ} \mathrm{C}$. The mutant showed hypersensitive response to exogenous abscisic acid and salinity during germination and during post-germinative growth (Sun et al., 2016). AtHSP24.7 has been described as a central activator of temperature-dependent seed germination (Ma et al., 2019). AtHSP24.7 overexpression accelerated seed germination and caused the accumulation of reactive oxygen species (ROS). In the study of $\mathrm{Ma}$ et al. (2019), an absence of negative physiology impact on plants was observed. Yet, it remains to demonstrate that other HSP20 family members behave similarly to HSP24.7, which could increase ROS content and, thereby, inducing apoptosis when achieving a critical threshold. Other related studies have indicated that the overaccumulation of HSP molecular chaperones might be deleterious for plants. For instance, although the overexpression of HSP70-1 improved basal thermotolerance in A. thaliana, it resulted in a dwarf phenotype, altering root growth (Sung and Guy, 2015). Furthermore, overexpression lines of HSP90.2, HSP90.5, and HSP90.7 reduced the resistance to salt and drought stress and produced a lower germination rate and lower fresh weight (Song et al., 2009). Thus, plants seem to have established a sophisticated mechanism to tightly regulate the expression of HSP chaperones, presumably to not affect plant fitness (Figure 2).

\section{CONCLUSION}

In recent years, the threat of global warming and the widereaching implications of the adverse effects on plant growth and

\section{REFERENCES}

Aldon, D., and Galaud, J. (2006). Plant Calmodulins and Calmodulin-Related Proteins. Plant Signal. Behav. 1, 96-104. doi: 10.4161/psb.1.3.2998

Al-Whaibi, M. H. (2011). Plant heat-shock proteins: a mini review. J. King Saud Univ. Sci. 23, 139-150. doi: 10.1016/j.jksus.2010.06.022

Anckar, J., and Sistonen, L. (2011). Regulation of HSF1 Function in the Heat Stress Response: implications in Aging and Disease. Annu. Rev. Biochem. 80, 1089-1115.

Basha, E., O'Neill, H., and Vierling, E. (2012). Small heat shock proteins and $\alpha$-crystallins: dynamic proteins with flexible functions. Trends Biochem. Sci. 37, 106-117. doi: 10.1016/j.tibs.2011.11.005 crop yields have called for more studies about HS. This review has described some aspects of the heat perception and molecules involved in the signaling, ultimately triggering the accumulation of protective HSPs. There is strong evidence in literature showing that the plasma membrane, embedded with CNGCs, acts as a central hub for the perception of incremental temperature. Yet, an unidentified signal, potentially involving calmodulins and kinases, triggers the translocation of HSFA1s into the nucleus to activate essential regulatory responses, such as histone and DNA regulation (Figure 1). To further investigate the heat shock signaling pathway, several questions remain unanswered; among them are the following:

- What are the missing partners involved in the heat signal transduction between CNGCs and the activation of HSFA1 upon HS?

- How are CNGCs subunits assembled to sense and respond to a wide temperature scale?

- Does the overexpression of HSP20s at low temperature induce deleterious phenotypes in land plants?

All these issues need further research to address a comprehensive picture of heat sensing and AT.

\section{AUTHOR CONTRIBUTIONS}

BB made the figures and AG has updated Figure 1. Both the authors conceived the central ideas of the manuscript, interpreted data from literature, contributed to writing, reviewed, edited, and approved its final version of the manuscript.

\section{FUNDING}

This work was supported and funded by the University of Lausanne and the Swiss National Fund grant $\mathrm{n}^{\circ} \mathrm{CRSK}-$ 3_196689 to AG.

\section{ACKNOWLEDGMENTS}

We would like to thank Elia Stahl, Robertas Ursache, Alexandra Waskow, and Pierre Goloubinoff for bringing precious pieces of advice and comments on this manuscript.

Bäurle, I., and Trindade, I. (2020). Chromatin regulation of somatic abiotic stress memory. J. Exp. Bot. 71, 5269-5279. doi: 10.1093/jxb/era a098

Ben-Zvi, A. P., and Goloubinoff, P. (2001). Review: mechanisms of disaggregation and refolding of stable protein aggregates by molecular chaperones. J. Struct. Biol. 135, 84-93.

Boyko, A., Blevins, T., Yao, Y., Golubov, A., Bilichak, A., Ilnytskyy, Y., et al. (2010). Transgenerational Adaptation of Arabidopsis to Stress Requires DNA Methylation and the Function of Dicer-Like Proteins. PLoS One 5:e9514. doi: 10.1371/journal.pone.0009514

Bussell, J. D., Estavillo, G. M., Che, P., Pogson, B. J., Smith, S. M., and Zhou, W. (2010). Signaling from the Endoplasmic Reticulum Activates Brassinosteroid 
Signaling and Promotes Acclimation to Stress in Arabidopsis. Sci. Signal. 3:ra69. doi: $10.1126 /$ scisignal.2001140

Chang, F., Ma, H., Yang, H., Song, Z.-T., Liu, J.-X., Ding, L., et al. (2017). TissueSpecific Transcriptomics Reveals an Important Role of the Unfolded Protein Response in Maintaining Fertility upon Heat Stress in Arabidopsis. Plant Cell 29, 1007-1023.

Chan-Schaminet, K. Y., Baniwal, S. K., Bublak, D., Nover, L., and Scharf, K. D. (2009). Specific interaction between tomato HsfA1 and HsfA2 creates heterooligomeric superactivator complexes for synergistic activation of heat stress gene expression. J. Biol. Chem. 284, 20848-20857. doi: 10.1074/jbc.M109. 007336

Chantarachot, T., and Bailey-Serres, J. (2018). Polysomes, stress granules, and processing bodies: a dynamic triumvirate controlling cytoplasmic mRNA fate and function. Plant Physiol. 176, 254-269. doi: 10.1104/pp.17.01468

Chauhan, H., Khurana, N., Nijhavan, A., Khurana, J. P., and Khurana, P. (2012). The wheat chloroplastic small heat shock protein (sHSP26) is involved in seed maturation and germination and imparts tolerance to heat stress. Plant Cell Environ. 35, 1912-1931.

Chodasiewicz, M., Sokolowska, E. M., Nelson-Dittrich, A., Masiuk, A., Beltran, J. C. M., Nelson, A., et al. (2020). Identification and characterization of the heat-induced plastidial stress granules reveal new insight into arabidopsis stress response. Front. Plant Sci. 11:595792. doi: 10.3389/fpls.2020.595792

Clough, S. J., Fengler, K. A., Yu, I. C., Lippok, B., Smith, R. K., and Bent, A. F. (2000). The Arabidopsis dnd1 "defense, no death" gene encodes a mutated cyclic nucleotide-gated ion channel. Proc. Natl. Acad. Sci. U. S. A. 97, 9323-9328.

Cortijo, S., Charoensawan, V., Brestovitsky, A., Buning, R., Ravarani, C., Rhodes, D., et al. (2017). Transcriptional Regulation of the Ambient Temperature Response by H2A.Z Nucleosomes and HSF1 Transcription Factors in Arabidopsis. Mol. Plant 10, 1258-1273. doi: 10.1016/j.molp.2017.08.014

Cui, Y., Lu, S., Li, Z., Cheng, J., Hu, P., Zhu, T., et al. (2020). CYCLIC NUCLEOTIDE-GATED ION CHANNELs 14 and 16 Promote Tolerance to Heat and Chilling in Rice. Plant Physiol. 183, 1794-1808.

Czarnecka-Verner, E., Yuan, C. X., Nover, L., Scharf, K. D., Englich, G., and Gurley, W. B. (1997). Plant heat shock transcription factors: positive and negative aspects of regulation. Acta Physiol. Plant 19, 529-537.

Demidchik, V., Shabala, S., Isayenkov, S., Cuin, T. A., and Pottosin, I. (2018). Calcium transport across plant membranes: mechanisms and functions. New Phytol. 220, 49-69. doi: 10.1111/nph.15266

Dong, B., Sutton, R. T., and Shaffrey, L. (2017). Understanding the rapid summer warming and changes in temperature extremes since the mid-1990s over Western Europe. Clim Dyn 48, 1537-1554. doi: 10.1007/s00382-016-3158-8

Finka, A., Cuendet, A. F. H., Maathuis, F. J. M., Saidi, Y., and Goloubinoff, P. (2012). Plasma Membrane Cyclic Nucleotide Gated Calcium Channels Control Land Plant Thermal Sensing and Acquired Thermotolerance. Plant Cell 24, 3333-3348. doi: 10.1105/tpc.112.095844

Finka, A., and Goloubinoff, P. (2014). The CNGCb and CNGCd genes from Physcomitrella patens moss encode for thermosensory calcium channels responding to fluidity changes in the plasma membrane. Cell Stress Chaperones 19, 83-90.

Finka, A., Mattoo, R. U. H., and Goloubinoff, P. (2011). Meta-analysis of heat-and chemically upregulated chaperone genes in plant and human cells. Cell Stress Chaperones 16, 15-31.

Finka, A., Sharma, S. K., and Goloubinoff, P. (2015). Multi-layered molecular mechanisms of polypeptide holding, unfolding and disaggregation by HSP70/HSP110 chaperones. Front. Mol. Biosci. 2:29. doi: 10.3389/fmolb.2015. 00029

Fischer, C., Defalco, T. A., Karia, P., Snedden, W. A., Moeder, W., Yoshioka, K., et al. (2017). Calmodulin as a Ca2+-Sensing Subunit of Arabidopsis Cyclic Nucleotide-Gated Channel Complexes. Plant Cell Physiol. 58, 1208-1221.

Fischer, C., Kugler, A., Hoth, S., and Dietrich, P. (2013). An IQ domain mediates the interaction with calmodulin in a plant cyclic nucleotide-gated channel. Plant Cell Physiol. 54, 573-584.

Fragkostefanakis, S., Roth, S., Scheleiff, E., and Scharf, K. D. (2015). Prospects of engineering thermotolerance in crops through modulation of heat stress transcription factor and heat shock protein networks. Plant Cell Environ. 38, 1881-1895. doi: 10.1111/pce.12396

Franklin, K. A. (2010). Plant Chromatin Feels the Heat. Cell 140, 26-28.
Gallego-Bartolomé, J. (2020). DNA methylation in plants: mechanisms and tools for targeted manipulation. New Phytologist 227, 38-44. doi: 10.1111/nph. 16529

Gallo, G. J., Prentice, H., and Kingston, R. E. (1993). Heat shock factor is required for growth at normal temperatures in the fission yeast Schizosaccharomyces pombe. Mol. Cell Biol. 13, 749-761.

Gao, F., Han, X., Wu, J., Zheng, S., Shang, Z., Sun, D., et al. (2012). A heat-activated calcium-permeable channel - Arabidopsis cyclic nucleotidegated ion channel 6 - Is involved in heat shock responses. Plant J. 70, 1056-1069.

Gao, G., Li, J., Li, H., Li, F., Xu, K., Yan, G., et al. (2014). Comparison of the heat stress induced variations in DNA methylation between heat-tolerant and heat-sensitive rapeseed seedlings. Breed. Sci. 64, 125-133. doi: 10.1270/jsbbs. 64.125

Glover, J. R., and Lindquist, S. (1998). Hsp104, Hsp70, and Hsp40: a novel chaperone system that rescues previously aggregated proteins. Cell 94, 73-82.

Goloubinoff, P., Mogk, A., Ben Zvi, A. P., Tomoyasu, T., and Bukau, B. (1999). Sequential mechanism of solubilization and refolding of stable protein aggregates by a bichaperone network. Proc. Natl. Acad. Sci. U. S. A. 96, $13732-13737$.

Gomez-Pastor, R., Burchfiel, E., and Thiele, D. (2018). Regulation of heat shock transcription factors and their roles in physiology and disease. Nat. Rev. Mol. Cell Biol. 19, 4-19. doi: 10.1038nrm.2017.73

Gong, M., Luit, A. H., Van Der, Knight, M. R., and Trewavas, A. J. (1998). HeatShock-Induced Changes in Intracellular $\mathrm{Ca}^{2+}$ Level in Tobacco Seedlings in Relation to Thermotolerance. Plant Physiol. 116, 429-437. doi: 10.1104/pp.116. 1.429

Guihur, A., Fauvet, B., Finka, A., Quadroni, M., and Goloubinoff, P. (2020). Quantitative proteomic analysis to capture the role of heat-accumulated proteins in moss plant acquired thermotolerance. Plant Cell Environ. 2020, $1-17$.

Guo, M., Liu, J.-H., Ma, X., Luo, D.-X., Gong, Z.-H., and Lu, M.-H. (2016). The plant Heat Stress Transcription Factors (HSFs): structure, regulation, and function in response to Abiotic stresses. Front. Plant Sci. 7:114. doi: 10.3389/ fpls.2016.00114

Hahn, A., Bublak, D., Schleiff, E., and Scharf, K. D. (2011). Crosstalk between Hsp90 and Hsp70 chaperones and heat stress transcription factors in tomato. Plant Cell 23, 741-755.

Haslbeck, M., Franzmann, T., Weinfurtner, D., and Buchner, J. (2005). Some like it hot: the structure and function of small heat-shock proteins. Nat. Struct. Mol. Biol. 12, 842-846.

Haslbeck, M., and Vierling, E. (2015). A first line of stress defense: small heat shock proteins and their function in protein homeostasis. J. Mol. Biol. 427, 1537-1548. doi: 10.1016/j.jmb.2015.02.002

Hayashida, N., Fujimoto, M., and Nakai, A. (2011). Transcription factor cooperativity with heat shock factor 1 . Transcription 2, 91-94. doi: 10.4161/trns. 2.2.14962

Hentze, N., Le Breton, L., Wiesner, J., Kempf, G., and Mayer, M. P. (2016). Molecular mechanism of thermosensory function of human heat shock transcription factor Hsf1. Elife 5:e11576.

Hernandez, L. D., and Vierling, E. (1993). Expression of low molecular weight heat-shock proteins under field conditions. Plant Physiol. 101, 1209-1216.

Horváth, I., Multhoff, G., Sonnleitner, A., and Vígh, L. (2008). Biochimica et Biophysica Acta Membrane-associated stress proteins?: more than simply chaperones. Biochim. Biophys. Acta 1778, 1653-1664. doi: 10.1016/j.bbamem. 2008.02.012

Huang, Y. C., Niu, C. Y., Yang, C. R., and Jinn, T. L. (2016). The heat-stress factor HSFA6b connects ABA signaling and ABA-mediated heat responses. Plant Physiol. 172, 1182-1199. doi: 10.1104/pp.16.00860

Ikeda, M., Mitsuda, N., and Ohme-Takagi, M. (2011). Arabidopsis HsfB1 and $\mathrm{HsfB} 2 \mathrm{~b}$ act as repressors of the expression of heat-inducible Hsfs but positively regulate the acquired thermotolerance. Plant Physiol. 157, 1243-1254. doi: 10. 1104/pp.111.179036

Jarratt-Barnham, E., Wang, L., Ning, Y., and Davies, J. M. (2021). The complex story of plant cyclic nucleotide-gated channels. Int. J. Mol. Sci. 22, 1-26.

Jee, H. (2016). Size dependent classification of heat shock proteins: a mini-review. J. Exerc. Rehabil. 12, 255-259. doi: 10.12965/jer.1632642.321 
Kaplan, B., Sherman, T., and Fromm, H. (2007). Cyclic nucleotide-gated channels in plants. FEBS Lett. 581, 2237-2246. doi: 10.1016/j.febslet.2007.02.017

Khraiwesh, B., Zhu, J. K., and Zhu, J. (2012). Role of miRNAs and siRNAs in biotic and abiotic stress responses of plants. Biochim. Biophys. Acta 1819, 137-148.

Kijima, T., Prince, T. L., Tigue, M. L., Yim, K. H., Schwartz, H., Beebe, K., et al. (2018). HSP90 inhibitors disrupt a transient HSP90-HSF1 interaction and identify a noncanonical model of HSP90-mediated HSF1 regulation. Sci. Rep. $8,1-13$.

Kim, B.-H., and Schöffl, F. (2002). Interaction between Arabidopsis heat shock transcription factor 1 and $70 \mathrm{kDa}$ heat shock proteins. J. Exp. Bot. 53, 371-375. doi: $10.1093 /$ jexbot $/ 53.367 .371$

Kosmacz, M., Gorka, M., Schmidt, S., Luzarowski, M., Moreno, J. C., Szlachetko, J., et al. (2019). Protein and metabolite composition of Arabidopsis stress granules. New Phytol. 222, 1420-1433. doi: 10.1111/nph.15690

Kumar, S. V., and Wigge, P. A. (2010). H2A.Z-Containing Nucleosomes Mediate the Thermosensory Response in Arabidopsis. Cell 140, 136-147. doi: 10.1016/j. cell.2009.11.006

Kyle Hadden, M., Lubbers, D., and Blagg, B. S. J. (2006). Geldanamycin, Radicicol, and Chimeric Inhibitors of the Hsp90 Nterminal ATP Binding Site. Curr. Top. Med. Chem. 6, 1173-1182.

Lämke, J., Brzezinka, K., Altmann, S., and Bäurle, I. (2016). A hit-and-run heat shock factor governs sustained histone methylation and transcriptional stress memory. EMBO J. 35, 162-175. doi: 10.15252/embj.201592593

Larkindale, J., Hall, J. D., Knight, M. R., and Vierling, E. (2005). Heat Stress Phenotypes of Arabidopsis Mutants Implicate Multiple Signaling Pathways in the Acquisition of Thermotolerance. Plant Physiol. 138, 882-897.

Lee, B. H., Won, S. H., Lee, H. S., Miyao, M., Chung, W. I., Kim, I. J., et al. (2000). Expression of the chloroplast-localized small heat shock protein by oxidative stress in rice. Gene 245, 283-290. doi: 10.1016/s0378-1119(00)00043-3

Li, B., Liu, H., Sun, D., and Zhou, R. (2004). $\mathrm{Ca}^{2+}$ and Calmodulin Modulate DNABinding Activity of Maize Heat Shock Transcription Factor in Vitro. Plant Cell Physiol. 45, 627-634. doi: 10.1093/pcp/pch074

Li, S., Liu, J., Liu, Z., Li, X., Wu, F., and He, Y. (2014). HEAT-INDUCED TAS1 TARGET1 mediates thermotolerance via heat stress transcription factor Aladirected pathways in Arabidopsis. Plant Cell 26, 1764-1780. doi: 10.1105/tpc. 114.124883

Li, Z. G., Ye, X. Y., and Qiu, X. M. (2019). Glutamate signaling enhances the heat tolerance of maize seedlings by plant glutamate receptor-like channelsmediated calcium signaling. Protoplasma 256, 1165-1169.

Liao, C., Zheng, Y., and Guo, Y. (2017). MYB30 transcription factor regulates oxidative and heat stress responses through ANNEXIN-mediated cytosolic calcium signaling in Arabidopsis. New Phytologist 216, 163-177. doi: 10.1111/ nph.14679

Liberek, K., Lewandowska, A., and Ziętkiewicz, S. (2008). Chaperones in control of protein disaggregation. ЕMBO J. 27, 328-335.

Lin, J. S., Kuo, C. C., Yang, I. C., Tsai, W. A., Shen, Y. H., Lin, C. C., et al. (2018). MicroRNA160 Modulates Plant Development and Heat Shock Protein Gene Expression to Mediate Heat Tolerance in Arabidopsis. Front. Plant Sci. 9:68. doi: $10.3389 /$ fpls.2018.00068

Lin, K. F., Tsai, M. Y., Lu, C. A., Wu, S. J., and Yeh, C. H. (2018). The roles of Arabidopsis HSFA2, HSFA4a, and HSFA7a in the heat shock response and cytosolic protein response. Bot. Stud. 59:15. doi: 10.1186/s40529-018-0231-0

Liu, H., Li, B., Shang, Z., Li, X., Mu, R., Sun, D., et al. (2003). Calmodulin Is Involved in Heat Shock Signal Transduction in Wheat. Plant Physiol. 132, 1186-1195.

Liu, H. C., and Charng, Y. Y. (2012). Acquired thermotolerance independent of heat shock factor A1 (HsfA1), the master regulator of the heat stress response. Plant Signal. Behav. 7, 1-5.

Liu, H. C., and Charng, Y. Y. (2013). Common and distinct functions of Arabidopsis class $\mathrm{A} 1$ and $\mathrm{A} 2$ heat shock factors in diverse abiotic stress responses and development. Plant Physiol. 163, 276-290.

Liu, H. C., Liao, H. T., and Charng, Y. Y. (2011). The role of class A1 heat shock factors (HSFA1s) in response to heat and other stresses in Arabidopsis. Plant Cell Environ. 34, 738-751.

Liu, H. T., Gao, F., Li, G. L., Han, J. L., Liu, D. L., Sun, D. Y., et al. (2008). The calmodulin-binding protein kinase 3 is part of heat-shock signal transduction in Arabidopsis thaliana. Plant J. 55, 760-773.
Liu, H. T., Sun, D. Y., and Zhou, R. G. (2005). $\mathrm{Ca}^{2+}$ and AtCaM3 are involved in the expression of heat shock protein gene in Arabidopsis. Plant Cell Environ. 28, 1276-1284.

Liu, Q., Yan, S., Yang, T., Zhang, S., Chen, Y. Q., and Liu, B. (2017). Small RNAs in regulating temperature stress response in plants. J. Integr. Plant Biol. 59, 774-791. doi: 10.1111/jipb.12571

Ma, W., Guan, X., Li, J., Pan, R., Wang, L., Liu, F., et al. (2019). Mitochondrial small heat shock protein mediates seed germination via thermal sensing. Proc. Natl. Acad. Sci. U. S. A. 116, 4716-4721.

Mangelsen, E., Kilian, J., Harter, K., Jansson, C., Wanke, D., and Sundberg, E. (2011). Transcriptome analysis of high-temperature stress in developing barley caryopses: early stress responses and effects on storage compound biosynthesis. Mol. Plant 4, 97-115. doi: 10.1093/mp/ssq058

March-Díaz, R., and Reyes, J. C. (2009). The beauty of being a variant: H2A.Z and the SWR1 complex in plants. Mol. Plant 2, 565-577. doi: 10.1093/mp/ssp019

Mccormack, E., Tsai, Y., and Braam, J. (2005). Handling calcium signaling : Arabidopsis CaMs and CMLs. Trends Plant Sci. 10, 383-389. doi: 10.1016/j. tplants.2005.07.00

McLoughlin, F., Basha, E., Fowler, M. E., Kim, M., Bordowitz, J., Katiyar-Agarwal, S., et al. (2016). Class I and II Small Heat Shock Proteins Together with HSP101 Protect Protein Translation Factors during Heat Stress. Plant Physiol. 172, 1221-1236. doi: 10.1104/pp.16.00536

McLoughlin, F., Kim, M., Marshall, R. S., Vierstra, R. D., and Vierling, E. (2019). HSP101 Interacts with the Proteasome and Promotes the Clearance of Ubiquitylated Protein Aggregates. Plant Physiol. 180, 1829-1847. doi: 10.1104/ pp.19.00263

Merret, R., Carpentier, M. C., Favory, J. J., Picart, C., Descombin, J., BousquetAntonelli, C., et al. (2017). Heat Shock Protein HSP101 Affects the Release of Ribosomal Protein mRNAs for Recovery after Heat Shock. Plant Physiol. 174, 1216-1225. doi: 10.1104/pp.17.00269

Mishra, S. K., Tripp, J., Winkelhaus, S., Tschiersch, B., Theres, K., Nover, L., et al. (2002). In the complex family of heat stress transcription factors, HsfA1 has a unique role as master regulator of thermotolerance in tomato. Genes Dev. 16, $1555-1567$.

Mitsuda, N., and Ohme-Takagi, M. (2009). Functional analysis of transcription factors in Arabidopsis. Plant Cell Physiol. 50, 1232-1248.

Mittler, R., Finka, A., and Goloubinoff, P. (2012). How do plants feel the heat? Trends Biochem. Sci. 37, 118-125. doi: 10.1016/j.tibs.2011.11.007

Mogk, A., and Bukau, B. (2017). Role of sHsps in organizing cytosolic protein aggregation and disaggregation. Cell Stress Chaperones 22, 493-502.

Mogk, A., Kummer, E., and Bukau, B. (2015). Cooperation of Hsp70 and Hsp100 chaperone machines in protein disaggregation. Front. Mol. Biosci. 2:22. doi: 10.3389/fmolb.2015.00022

Mogk, A., Schlieker, C., Friedrich, K. L., Schönfeld, H. J., Vierling, E., and Bukau, B. (2003). Refolding of substrates bound to small Hsps relies on a disaggregation reaction mediated most efficiently by $\mathrm{ClpB} / \mathrm{DnaK}$. J. Biol. Chem. 278, 3103331042.

Morimoto, R. I. (2012). The heat shock response : systems biology of proteotoxic stress in aging and disease. Cold Spring Harb. Symp. Quant. Biol. 76, 91-99.

Nicholls, S., Leach, M. D., Priest, C. L., and Brown, A. J. P. (2009). Role of the heat shock transcription factor, Hsfl, in a major fungal pathogen that is obligately associated with warm-blooded animals. Mol. Microbiol. 74, 844-861.

Niu, W. T., Han, X. W., Wei, S. S., Shang, Z. L., Wang, J., Yang, D. W., et al. (2020). Arabidopsis cyclic nucleotide-gated channel 6 is negatively modulated by multiple calmodulin isoforms during heat shock. J. Exp. Bot. 71, 90-114.

Nover, L., Bharti, K., Döring, P., Mishra, S. K., Ganguli, A., and Scharf, K. D. (2001). Arabidopsis and the heat stress transcription factor world: how many heat stress transcription factors do we need? Cell Stress Chaperones 6, 177-189. doi: 10.1379/1466-1268(2001)006<0177:aathst $<2.0$. co;2

Ohama, N., Sato, H., Shinozaki, K., and Yamaguchi-Shinozaki, K. (2017). Transcriptional Regulatory Network of Plant Heat Stress Response. Trends Plant Sci. 22, 53-65. doi: 10.1016/j.tplants.2016.08.015

Probst, A. V., and Mittelsten Scheid, O. (2015). Stress-induced structural changes in plant chromatin. Curr. Opin. Plant Biol. 27, 8-16. doi: 10.1016/j.pbi.2015. 05.011

Qin, D., Wu, H., Peng, H., Yao, Y., Ni, Z., Li, Z., et al. (2008). Heat stressresponsive transcriptome analysis in heat susceptible and tolerant wheat 
(Triticum aestivum L.) by using Wheat Genome Array. BMC Genomics 9:432. doi: 10.1186/1471-2164-9-432

Rhoads, A. R., and Friedberg, F. (1997). Sequence motifs for calmodulin recognition. FASEB J. 11, 331-340.

Saidi, Y., Finka, A., Muriset, M., Bromberg, Z., Weiss, Y. G., Maathuis, F. J. M., et al. (2009). The Heat Shock Response in Moss Plants Is Regulated by Specific Calcium-Permeable Channels in the Plasma Membrane. Plant Cell Online 21, 2829-2843. doi: $10.1105 /$ tpc. 108.065318

Santoro, N., Johansson, N., and Thiele, D. J. (1998). Heat Shock Element Architecture Is an Important Determinant in the Temperature and Transactivation Domain Requirements for Heat Shock Transcription Factor. Mol. Cell Biol. 18, 6340-6352.

Santos, A. P., Ferreira, L., Maroco, J., and Oliveira, M. M. (2011). Abiotic stress and induced DNA hypomethylation cause interphase chromatin structural changes in rice rDNA loci. Cytogenet. Genome Res. 132, 297-303.

Sarkar, N. K., Kim, Y., and Grover, A. (2009). Rice sHsp genes : genomic organization and expression profiling under stress and development. BMC Genomics 10:393. doi: 10.1186/1471-2164-10-393

Scharf, K. D., Berberich, T., Ebersberger, I., and Nover, L. (2012). The plant heat stress transcription factor (Hsf) family: structure, function and evolution. Biochim. Biophys. Acta 1819, 104-119. doi: 10.1016/j.bbagrm.2011.10.002

Schmollinger, S., Schulz-Raffelt, M., Strenkert, D., Veyel, D., Vallon, O., and Schroda, M. (2013). Dissecting the heat stress response in chlamydomonas by pharmaceutical and RNAi approaches reveals conserved and novel aspects. Mol. Plant 6, 1795-1813.

Schwarzländer, M., and Finkemeier, I. (2012). Mitochondrial Energy and Redox Signaling in Plants. Antioxid. Redox. Signal. 18, 2122-2144.

Serrano, N., Ling, Y., Bahieldin, A., and Mahfouz, M. M. (2019). Thermopriming reprograms metabolic homeostasis to confer heat tolerance. Sci. Rep. 9:181.

Simões-Araújo, J. L., Rumjanek, N. G., and Margis-Pinheiro, M. (2003). Small heat shock proteins genes are differentially expressed in distinct varieties of common bean. Braz. J. Plant Physiol. 15, 33-41.

Song, H., Zhao, R., Fan, P., Wang, X., Chen, X., and Li, Y. (2009). Overexpression of AtHsp90.2, AtHsp90.5 and AtHsp90.7 in Arabidopsis thaliana enhances plant sensitivity to salt and drought stresses. Planta 229, 955-964. doi: 10.1007/ s00425-008-0886.-y

Song, L., Jiang, Y., Zhao, H., and Hou, M. (2012). Acquired thermotolerance in plants. Plant Cell Tissue Organ Cult. 111, 265-276.

Song, N., and Ahn, Y. (2010). DcHsp17.7, a small heat shock protein from carrot, is upregulated under cold stress and enhances cold tolerance by functioning as a molecular chaperone. Hortscience 45, 469-474.

Sun, A.-Z., and Guo, F.-Q. (2016). Chloroplast retrograde regulation of heat stress responses in plants. Front. Plant Sci. 7:398. doi: 10.3389/fpls.2016.00398

Sun, W., Montagu, M. V., and Verbruggen, N. (2002). Small heat shock proteins and stress tolerance in plants. Biochim. Biophys. Acta 1577, 1-9.

Sun, X., Sun, C., Li, Z., Hu, Q., Han, L., and Luo, H. (2016). AsHSP17, a creeping bentgrass small heat shock protein modulates plant photosynthesis and ABAdependent and independent signalling to attenuate plant response to abiotic stress. Plant Cell Environ. 39, 1320-1337. doi: 10.1111/pce.12683

Sung, D. Y., and Guy, C. L. (2015). Physiological and Molecular Assessment of Altered Expression of Hsc70-1 in Arabidopsis. Evidence for Pleiotropic Consequences. Plant Physiol. 132, 979-987. doi: 10.1104/pp.102.019398

Suzuki, N., Sejima, H., Tam, R., Schlauch, K., and Mittler, R. (2011). Identification of the MBF1 heat-response regulon of Arabidopsis thaliana. Plant J. 66, 844851. doi: 10.1111/j.1365-313X.2011.04550.x

Swindell, W. R., Huebner, M., and Weber, A. P. (2007). Transcriptional profiling of Arabidopsis heat shock proteins and transcription factors reveals extensive overlap between heat and non-heat stress response pathways. BMC Genomics 8:125. doi: 10.1186/1471-2164-8-125

Tan, Y.-Q., Yang, Y., Zhang, A., Fei, C.-F., Gu, L.-L., Sun, S.-J., et al. (2020). Three CNGC family members, CNGC5, CNGC6, and CNGC9, are required for constitutive growth of Arabidopsis root hairs as Ca2+-Permeable Channels. Plant Commun. 1:100001.

Tomás, D., Brazão, J., Viegas, W., and Silva, M. (2013). Differential effects of high-temperature stress on nuclear topology and transcription of repetitive noncoding and coding rye sequences. Cytogenet. Genome Res. 139, 119-127.
Tunc-Ozdemir, M., Tang, C., Ishka, M. R., Brown, E., Groves, N. R., Myers, C. T., et al. (2013). A cyclic nucleotide-gated channel (CNGC16) in pollen is critical for stress tolerance in pollen reproductive development. Plant Physiol. 161, 1010-1020.

Vadassery, J., Scholz, S. S., and Mithöfer, A. (2012). Multiple calmodulin-like proteins in Arabidopsis are induced by insect-derived (Spodoptera littoralis) oral secretion. Plant Signal. Behav. 7, 1277-1280.

Veinger, L., Diamant, S., Buchner, J., and Goloubinoff, P. (1998). The small heatshock protein $\mathrm{IbpB}$ from Escherichia coli stabilizes stress-denatured proteins for subsequent refolding by a multichaperone network. J. Biol. Chem. 273, $11032-11037$.

Véry, A. A., and Sentenac, H. (2002). Cation channels in the Arabidopsis plasma membrane. Trends Plant Sci. 7, 168-175.

Vierling, E. (2003). The Roles of Heat Shock Proteins in Plants. Ann. Rev. Plant Biol. 42, 579-620. doi: 10.1146/annurev.pp.42.060191.003051

Virdi, A. S., Singh, S., and Singh, P. (2015). Abiotic stress responses in plants: roles of calmodulin-regulated proteins. Front. Plant Sci. 2015:809. doi: 10.3389/fpls. 2015.00809

Volkov, R. A., Panchuk, I. I., and Scho, F. (2005). Small heat shock proteins are differentially regulated during pollen development and following heat stress in tobacco. Plant Mol. Biol. 57, 487-502. doi: 10.1007/s11103-005-0339-y

von Koskull-Doring, P., Scharf, K. D., and Nover, L. (2007). The diversity of plant heat stress transcription factors. Trends Plant Sci.Rev. 12, 452-457. doi: 10.1016/j.tplants.2007.08.014

Wang, W., Vinocur, B., Shoseyov, O., and Altman, A. (2004). Role of plant heatshock proteins and molecular chaperones in the abiotic stress response. Trends Plant Sci. 9, 244-252.

Waters, E. R., and Vierling, E. (2020). Plant small heat shock proteins evolutionary and functional diversity. New Phytologist 227, 24-37. doi: 10.1111/ nph.16536

Waters, E. R., Lee, G. J., and Vierling, E. (1996). Evolution, structure and function of the small heat shock proteins in plants. J. Exp. Bot. 47, 325-338. doi: 10.1093/ jxb/47.3.325

Westerheide, S. D., Kawahara, T. L. A., Orton, K., and Morimoto, R. I. (2006). Triptolide, an inhibitor of the human heat shock response that enhances stress-induced cell death. J. Biol. Chem. 281, 9616-9622.

Wu, H., and Jinn, T. (2010). Heat shock-triggered Ca2+ mobilization accompanied by pectin methylesterase activity and cytosolic $\mathrm{Ca} 2+$ oscillation are crucial for plant thermotolerance. Plant Signal. Behav. 5, 1252-1256. doi: 10.4161/psb.5. 10.12607

Wu, H. C., and Jinn, T. L. (2012). Oscillation regulation of Ca2+/calmodulin and heat-stress related genes in response to heat stress in rice (Oryza sativa L.). Plant Signal. Behav. 7, 1056-1057.

Wu, H. C., Luo, D. L., Vignols, F., and Jinn, T. L. (2012). Heat shockinduced biphasic $\mathrm{Ca}^{2+}$ signature and OsCaM1-1 nuclear localization mediate downstream signalling in acquisition of thermotolerance in rice (Oryza sativa L.). Plant Cell Environ. 35, 1543-1557. doi: 10.1111/j.1365-3040.2012. 02508.x

Xin, C., Wang, X., Cai, J., Zhou, Q., Liu, F., Dai, T., et al. (2016). Changes of transcriptome and proteome are associated with the enhanced post-anthesis high temperature tolerance induced by pre-anthesis heat priming in wheat. Plant Growth Regul. 79, 135-145.

Xue, G. P., Sadat, S., Drenth, J., and McIntyre, C. L. (2014). The heat shock factor family from Triticum aestivum in response to heat and other major abiotic stresses and their role in regulation of heat shock protein genes. J. Exp. Bot. 65, 539-557. doi: 10.1093/jxb/ert399

Yamada, K., Fukao, Y., Hayashi, M., Fukazawa, M., Suzuki, I., and Nishimura, M. (2007). Cytosolic HSP90 regulates the heat shock response that is responsible for heat acclimation in Arabidopsis thaliana. J. Biol. Chem. 282, 37794-37804.

Yamaguchi, N., Matsubara, S., Yoshimizu, K., Seki, M., Hamada, K., Kamitani, M., et al. (2021). H3K27me3 demethylases alter HSP22 and HSP17.6C expression in response to recurring heat in Arabidopsis. Nat. Commun. 12:3480. doi: 10.1038/ s41467-021-23766-w

Yan, Q., Huang, Q., Chen, J., Li, J., Liu, Z., Yang, Y., et al. (2017). SYTA has positive effects on the heat resistance of Arabidopsis. Plant Growth Regul. 81, 467-476.

Yang, G., Wang, Y., and Zhang, K. (2014). Expression analysis of nine small heat shock protein genes from Tamarix hispida in response to different abiotic 
stresses and abscisic acid treatment. Mol Biol Rep. 41, 1279-1289. doi: 10.1007/ s11033-013-2973-9

Yang, R., Yu, G., Li, H., Li, X., and Mu, C. (2020). Overexpression of Small Heat Shock Protein LimHSP16.45 in Arabidopsis hsp17.6II Mutant Enhances Tolerance to Abiotic Stresses. Russ. J. Plant Physiol. 67, 231-241.

Yip Delormel, T., and Boudsocq, M. (2019). Properties and functions of calciumdependent protein kinases and their relatives in Arabidopsis thaliana. New Phytol. 224, 585-604.

Yokotani, N., Ichikawa, T., Kondou, Y., Matsui, M., Hirochika, H., Iwabuchi, M., et al. (2008). Expression of rice heat stress transcription factor OsHsfA2e enhances tolerance to environmental stresses in transgenic Arabidopsis. Planta 227, 957-967. doi: 10.1007/s00425-007-0670-4

Yoshida, T., Ohama, N., Nakajima, J., Kidokoro, S., Mizoi, J., Nakashima, K., et al. (2011). Arabidopsis HsfAl transcription factors function as the main positive regulators in heat shock-responsive gene expression. Mol. Genet. Genomics 286, 321-332.

Zeng, X. C., Bhasin, S., Wu, X., Lee, J. G., Maffi, S., Nichols, C. J., et al. (2004). Hsp70 dynamics in vivo: effect of heat shock and protein aggregation. J. Cell Sci. 117, 4991-5000.

Zhang, J., Chen, H., Wang, H., Li, B., Yi, Y., Kong, F., et al. (2016). Constitutive Expression of a Tomato Small Heat Shock Protein Gene LeHSP21 Improves Tolerance to High-Temperature Stress by Enhancing Antioxidation Capacity in Tobacco. Plant Mol. Biol. Rep. 34, 399-409. doi: 10.1007/s11105-0150925-3

Zhang, S., Xu, Z. S., Li, P., Yang, L., Wei, Y., Chen, M., et al. (2013). Overexpression of TaHSF3 in Transgenic Arabidopsis Enhances Tolerance to Extreme Temperatures. Plant Mol. Biol. Rep. 31, 688-697. doi: 10.1007/s11105012-0546-z
Zhang, W., Zhou, R. G., Gao, Y. J., Zheng, S. Z., Xu, P., Zhang, S. Q., et al. (2009). Molecular and genetic evidence for the key role of AtCaM3 in heat-shock signal transduction in Arabidopsis1. Plant Physiol. 149, 1773-1784.

Zhao, J., He, Q., Chen, Q., Wang, L., and Jin, B. (2016). Regulation of Noncoding RNAs in Heat Stress Responses of Plants. Front. Plant Sci. 7:1213. doi: $10.3389 /$ fpls.2016.01213

Zhao, J., Lu, Z., Wang, L., and Jin, B. (2021). Plant responses to heat stress: physiology, transcription, noncoding RNAs, and epigenetics. Int J Mol Sci. 22:117. doi: 10.3390/ijms22010117

Zou, J., Guo, Y., Guettouche, T., Smith, D. F., and Voellmy, R. (1998). Repression of heat shock transcription factor HSF1 activation by HSP90 (HSP90 complex) that forms a stress-sensitive complex with HSF1. Cell 94, 471-480.

Conflict of Interest: The authors declare that the research was conducted in the absence of any commercial or financial relationships that could be construed as a potential conflict of interest.

Publisher's Note: All claims expressed in this article are solely those of the authors and do not necessarily represent those of their affiliated organizations, or those of the publisher, the editors and the reviewers. Any product that may be evaluated in this article, or claim that may be made by its manufacturer, is not guaranteed or endorsed by the publisher.

Copyright (C) 2021 Bourgine and Guihur. This is an open-access article distributed under the terms of the Creative Commons Attribution License (CC BY). The use, distribution or reproduction in other forums is permitted, provided the original author(s) and the copyright owner(s) are credited and that the original publication in this journal is cited, in accordance with accepted academic practice. No use, distribution or reproduction is permitted which does not comply with these terms. 